presented at a 2003 annual meeting of the Infantile Seizure Society in Tokyo, organized by Dr Yukio Fukuyama and colleagues (see Preface. Brain Dev March 2005;27:79).

\title{
NEUROLOGIC AND EEG FINDINGS IN ANGELMAN SYNDROME
}

The neurological and diagnostic aspects of Angelman syndrome (AS) are reviewed by a geneticist at the University of Florida, Gainesville, FL. The prevalence of AS is $1 / 10,000$ to $1 / 20,000$. The syndrome presents in infancy with global developmental delay, microcephaly, seizures or an ataxic/hypotonic form of cerebral palsy. The facial features and general physical examination are generally normal, although a protruding tongue, strabismus, brisk deep tendon reflexes, and a happy demeanor may be present. Hypopigmentation in infants with AS due to deletion of the P pigment gene but may be overlooked. The diagnosis becomes more evident after 1 or 2 years of age, when speech does not develop, walking is impaired by severe ataxia, and seizures occur. The EEG findings can be diagnostic, with high voltage slow waves at $4-6 \mathrm{c} / \mathrm{s}$ throughout the record, $2-3 \mathrm{c} / \mathrm{s}$ slow activity in runs, especially anteriorly, and spikes or sharp waves posteriorly, provoked by eye-closure. The diagnosis is usually obvious clinically after 3 years of age and is sometimes first suggested by the parents. Behavior is often outgoing, hyperactive, hyperexcitable with excessive laughing, grabbing to engage siblings, putting objects in the mouth, and drooling. These characteristics without signs of degeneration and associated with microcephaly, seizures, and ataxia are classical. Maternally derived chromosome 15 was implicated in 1980, with microdeletion of 15q11.215q13. Subsequently, the ubiquitin ligase gene, UBE3A, located at 15q11.2, was identified as the AS gene, and 4 genetic mechanisms were involved, microdeletions being most common. The type of genetic mechanism was correlated with the severity of AS, patients with large chromosome deletions having a greater risk of seizures, microcephaly, and hypopigmentation of skin, eye and hair. The distinct behavioral syndrome and seizure patterns are related to the effects of UBE3A occurring during neuronal development. DNA methylation testing of blood is a sensitive and specific screening for 3 of the 4 genetic mechanisms. Chromosome 15 FISH analysis is necessary to distinguish which mechanism is involved. In $10-15 \%$ of cases genetic test confirmation is not possible. Genetic counseling should be offered to families of AS patients since UBE3A mutations carry a $50 \%$ recurrence risk, while common deletion cases have $<1 \%$ recurrence risk. (Williams CA. Neurological aspects of the Angelman syndrome. Brain Dev March 2005;27:88-94). (Respond: Dr Charles A Williams, Division of Genetics, Department of Pediatrics, University of Florida, PO Box 100296, Gainesville, FL 32610).

COMMENT. Clinical consensus criteria for the diagnosis of AS (Williams CA, Angelman H, Clayton-Smith J et al. Am J Med Genet 1995;56:237-238) include consistent $(100 \%)$, frequent (in more than $80 \%$ ), and associated $(20-80 \%)$ symptoms and signs. The consistent findings include: developmental delay, speech impairment, ataxia or tremor, and unique behavioral abnormalities, with frequent laughing, happy demeanor, excitability, hand flapping, hyperactivity, and short attention span. Frequent findings include: delayed head growth with microcephaly by 2 years, seizures with onset $<3$ years, and characteristic abnormal EEG. Associated findings include: flat occiput, protruding tongue, feeding and swallowing disorders, drooling, strabismus, hypopigmented skin, hair and eyes, hyperactive relexes, flexed arm posture, sleep disorder, and fascination with water. 
The prevalence of EEG abnormalities in AS is $80 \%$, and 3 main patterns are present, independent of epileptic seizures: persistent rhythmic $4-6 \mathrm{~Hz}$ activity, anterior delta activity of 2-3 Hz with superimposed spikes and sharp waves, and posterior 3-4 Hz high amplitude waves with spikes and sharp waves, especially on eye closure. AS patients with a deletion of chromosome 15q11-13 have more prominent EEG abnormalities than those with other genetic disorders of chromosome 15 region. EEG abnormalities are helpful in the diagnosis of AS at an early age. (Laan LAEM, Vein AA. Brain Dev 2005;27:80-87).

The severity of developmental disturbance in AS is not invariably related to the severity of epilepsy, although repetitive nonconvulsive status epilepticus can sometimes result in transient or permanent mental and motor deterioration. (Ohtsuka Y et al. Brain Dev 2005;27:95-100).

\section{NONFASTING VERSUS INITIAL FASTING KETOGENIC DIET}

A retrospective evaluation of the ketogenic diet $(\mathrm{KD})$ was conducted comparing efficacy and tolerability of the diet with or without initial fasting and fluid restriction and involving university centers in Seoul, Korea. Of 41 children with intractable epilepsy treated with the nonfasting diet (NFKD), 14 (34.1\%) were seizure-free for at least 3 months, and in 83 receiving the initial fasting diet (IFKD), 29 (34.9\%) were seizure free. The patients' mean age was $4.1(+/-1.5)$ years in the NFKD group and $5.3(+/-1.6)$ years in the IFKD group. The incidence of various seizure types was not significantly different in the 2 groups. Strong urinary ketosis developed within $2.4+/-1.2$ days in the NFKD group, compared to $1.9+/-$ 1.3 days in the IFKD group (not significant, $\mathrm{P}>0.05$ ). Among early complications, dehydration was significantly less frequent in the NFKD group (12.2\%) than in the IFKD group $(62.7 \% ; \mathrm{P}<0.05)$. The incidence of other complications was not significantly different in the NF and IF groups, including hypoglycemia (4.9 v 4.8\%), nausea and vomiting ( $36 \mathrm{v}$

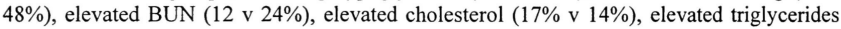
(36 v 36\%). Patients in the NFKD group were hospitalized for a mean period of $5.8(+/-2.7)$ days and those in the IFKD group for $7.8(+/-1.7)$ days $(\mathrm{P}<0.05)$. (Kim DW, Kang HC, Park JC, Kim HD. Benefits of the nonfasting ketogenic diet compared with the initial fasting ketogenic diet. Pediatrics Dec 2004;114:1627-1630). (Reprints: HD Kim MDPhD, Department of Pediatrics, Severance Hospital, Yonsei University College of Medicine, 134, Shinchon-dong, Seodaemun-gu, Seoul 120-752, Korea).

C OMMENT. The nonfasting ketogenic diet, in the above retrospective study, was of equal efficacy, and its introduction was better tolerated than the initial fasting method. The $\mathrm{KD}$ was first introduced for the treatment of epilepsy at the Mayo Clinic (Wilder RM. Mayo Clin Bull 1921;2:307). In the Mayo Clinic method, unlike the later Johns Hopkins protocol (Livingston S. Postgrad Med 1951;10:333-336), the $\mathrm{KD}$ is introduced without initial fasting and usually, without admission to hospital. The ratio of ketogenic to antiketogenic (K:AK) items in the diet is reversed gradually, starting at a ratio of 1:1.1 on day 1 and increasing to $2.8: 1$ by the $4^{\text {th }}$ day, or until ketones are found in the urine. In young children, a ratio of $3 ; 1$ is usually sufficient to maintain ketosis, and generally the higher $4: 1$ ratio is necessary only in older children. In my experience with the diet, beginning at the Mayo Clinic in the early 1960s and continuing at Children's Memorial Hospital in Chicago, the NFKD method has 